\title{
Genetic Factors and Psoriatic Arthritis
}

\author{
Monica Greco, Simone Negrini, Chiara Schiavi, Francesca Giusti, Matteo Borro, \\ Francesco Puppo, Giuseppe Murdaca*
}

Clinical Immunology Unit, Department of Internal Medicine, University of Genoa and Ospedale Policlinico San Martino,

Genoa, Italy

Email: ^giuseppe.murdaca@unige.it

How to cite this paper: Greco, M., Negrini, S., Schiavi, C., Giusti, F., Borro, M., Puppo, F. and Murdaca, G. (2019) Genetic Factors and Psoriatic Arthritis. Open Journal of Rheumatology and Autoimmune Diseases, 9, 111-120.

https://doi.org/10.4236/ojra.2019.93010

Received: May 9, 2019

Accepted: August 26, 2019

Published: August 29, 2019

Copyright (c) 2019 by author(s) and Scientific Research Publishing Inc. This work is licensed under the Creative Commons Attribution International License (CC BY 4.0).

http://creativecommons.org/licenses/by/4.0/

\begin{abstract}
Psoriatic arthritis (PsA) is a complex immune-mediated disease and its pathogenesis depends both on genetic factors and environment. PsA patients may present a wide range of clinical manifestations including skin and nail abnormalities. Indeed, articular involvement is variable too. Disease development relies on a heterogeneous net made of multiple cytokines pathways which are regulated by several factors including human leucocyte antigen (HLA) expression, miRNAs, microbiome. Among genetic polymorphisms which can lead to abnormal cytokine expression, tumor necrosis factor (TNF) polymorphisms have been studied. Thus, leading to the development of new therapeutic agents. Finally, further studies on genetic factors and epigenetics will give new insights into this complex disorder. The aim of this mini-review is to provide the reader with a summary of the fundamental and most innovative aspects of genetic and epigenetic factors involved in the PsA, thus including human leucocyte antigen (HLA) expression, tumor necrosis factor (TNF) polymorphisms, micro RNAs and microbiome.
\end{abstract}

\section{Keywords}

Psoriatic Arthritis, Psoriasis, HLA Associations, Genetic Heterogeneity, Phenotypic Heterogeneity, Epigenetics, Micro RNA, Microbiome,

TNF Polymorphisms

\section{Introduction}

Psoriatic arthritis (PsA) is a complex immune-mediated disease which occurs in $30 \%$ of patients suffering from psoriasis [1]. Although interest in musculoskeletal involvement among psoriatic (Ps) subjects has raised during the last decade, this condition is still underdiagnosed, thus leading to worse prognosis and decreased quality of life (QoL). PsA patients may present a wide range of clinical 
manifestations including skin and nail abnormalities. Articular involvement is variable. Indeed, according to Moll and Wright classification, there are five major phenotypes: symmetrical polyarthritis, asymmetrical oligoarthritis, distal interphalangeal (DIP) joint involvement, axial disease, and arthritis mutilans [2]. Due to the lack of specific biomarkers, PsA diagnosis is based on clinical and imaging characteristics. Considering the different timing of articular and skin involvement, a multidisciplinary approach is essential to avoid diagnosis delay. Diagnostic criteria for PsA have not been validated, but the Classification Criteria for PsA (CASPAR criteria), published in 2006, define psoriatic arthritis for the purpose of enrolling patients in clinical trials and provide guidance to clinicians [1]. Comorbidities are frequently reported among PsA patients including depression, cardiovascular disease (CVD), hypertension, diabetes/insulin resistance, metabolic syndrome, dyslipidemia, nonalcoholic fatty liver disease (NAFLD), obesity, inflammatory bowel disease, iridocyclitis, monoclonal gammopathy, and diffuse skeletal hyperostosis [3]. Although the pathogenesis of PsA remains elusive, It is now widely accepted that several factors may have a potential role in the pathogenesis of PsA including angiogenesis, inflammatory cytokines and viral infection (i.e., Epstein-Barr virus) [4]. Recent studies on regulatory lymphocytes demonstrated that CD8+ T suppressor (Ts) cells may have great relevance in controlling immune system homeostasis and avoiding the development of chronic inflammatory immune-mediated diseases. Among the three subpopulations of CD8+ Ts cells so far recognized in humans, the type 2 (non-antigen-specific) cell is characterized by the capacity to inhibit both $\mathrm{T}$ cell proliferation and cytotoxic $\mathrm{T}$ lymphocyte activity through secretion of soluble factors. It has been reported that type $2 \mathrm{CD} 8+$ Ts cells may be involved in the control of pathologic chronic immune responses, contributing in some cases to the pathogenesis of the inflammatory immune-mediated diseases including PsA [5]. PsA is considered a multifactorial disease in which both genetic and environmental factors may contribute. Notably, during the last years, epigenetic factors have also been studied [6].

As the pathogenesis of PsA relies on several mechanisms, during the last years different therapeutic strategies have been spread out. NSAIDS and traditional disease-modifying antirheumatic drugs (DMARDs) were the first agents to be used to treat this disease. However, they have been proved to have a limited effect on joint damage progression. Therefore, new agents as anti-TNF agents, including etanercept, infliximab, adalimumab, golimumab and certolizumab, as well as the newest molecules as oral phosphodiesterase 4 inhibitor (apremilast), Janus kinase (JAK) inhibitor (tofacitinib) and anti-IL-23/IL-17 pathway including secukinumab, brodalumab, ixekizumab, and ustekinumab have been developed.

\section{Family History}

PsA has a high heritability [7]. Indeed, family history of Ps has great relevance 
on PsA pathogenesis. In fact, according to CASPAR criteria, the presence of Ps in a first- or second-degree relative according to the patient stands as a diagnostic benchmark. Chandran et al. [8] conducted a study on 100 patients aiming to evaluate the recurrence risk of PsA and uncomplicated Ps in first-degree relatives (FDRs) of patients with PsA. They found that the recurrence risk ratio for both PsA and Ps is high in FDRs and siblings of patients with PsA. These results confirmed that both PsA and Ps have a strong heritable component. These results were also recently confirmed by Psoriasis Longitudinal Assessment and Registry (PSOLAR) [9]. Indeed, a family history of Ps was reported by $45.5 \%$ of patients, while $10.3 \%$ noted a family history of PsA.

\section{Human Leucocyte Antigen}

As PsA is a proteiform disease, different researchers focused on the genetic factors involved in PsA development. Similarly, to other models of chronic arthritis, like ankylosing spondylitis (AS), human leucocyte antigen (HLA) expression has been evaluated. However, it is widely accepted that AS is associated with HLA-B27 expression. PsA genetic background seems to be more complex. Roudier et al. [10] observed that among Ps patients presenting with the only axial disease, 57\% of them were positive for HLA-B27. On the other hand, regarding peripheral PsA one-third of patients expressed HLA-C06, one-fifth expressed HLA-B35, and one-tenth expressed HLA-B8. Each of these markers was associated with an odds ratio (OR) of 2 for developing PsA. The relevance of HLA-C06 upon Ps susceptibility was also confirmed by Tsoi et al. [11]. They conducted a meta-analysis on 10,588 cases and 22,806 controls including 3 genome-wide association studies (GWAS) and 2 independent data sets genotyped on the Immunochip, concluding that HLA C06 counted for more than $6 \%$ of the variance in disease risk. As previously mentioned, PsA can be considered an immune-mediated disease caused by the dysregulation of the innate and adaptive immune system, leading to skin and joint damage. ERAP is an aminopeptidase, which is highly active on immune cells and it takes part in the inflammatory pathway by cleaving cytokine receptors. Furthermore, ERAP allows proteins to be cleaved into small peptides that can be recognized by the immune system through the action of major histocompatibility complex (MHC) class I proteins. On these bases, Strange et al. [12] demonstrated that among Ps patients, there was a strong interaction between HLA-C and ERAP. Indeed, ERAP variants only influenced Ps susceptibility in individuals carrying the HLA-C risk allele. These results were also recently confirmed by Das et al. [13]. Several authors investigated on HLA-C06 and different clinical presentation of Ps. Fan et al. [14] conducted a study on Chinese Ps population demonstrating that HLA-C06 positive patients had an earlier disease onset, more severe disease, higher incidence of Köbner's phenomenon. HLA-C alleles are very close to the HLA-B alleles, therefore the HLA-C06 allele is often inherited with one of three HLA-B alleles: HLA-B ${ }^{\star} 57$, HLA- $B * 37$ and HLA- ${ }^{\star}{ }^{\star} 13$. These three allele combinations are often 
referred to as ancestral haplotypes [15]. Indeed, Gudjonsson et al. [16] conducted a study on 1019 patients with chronic plaque Ps demonstrating that the three ancestral haplotypes of HLA $\mathrm{Cw}^{\star} 0602$ all conferred an increase in odds ratio. However, they showed no difference in any of the clinical features studied. HLA-C06 expression was also studied with regards to therapy response. However, several drugs have been proposed to treat PsA, including highly effective monoclonal antibodies $(\mathrm{mAb})$ therapies such as adalimumab which targets tumor necrosis factor alpha (TNF- $\alpha$ ) and ustekinumab which binds to $\mathrm{p} 40$ subunit in order to inhibit IL 17 pathway. A large observational study, conducted by Dand et al. [17] found that HLA-C06-negative patients were significantly more likely to respond to adalimumab than ustekinumab and the difference was greater in HLA-C ${ }^{\star} 06: 02$ negative patients with PsA. Biologic-naive patients who were HLA-C06 positive and PsA negative demonstrated a significantly poorer response to adalimumab.

\section{TNF Polymorphisms}

Chronic inflammatory immune-mediated diseases are often associated with genetic polymorphisms which can lead to abnormal cytokine expression. During the last decades, researchers also focused on the role of tumor necrosis factor (TNF) and interleukin (IL) 17A and IL17F genes. These cytokines are encoded on the same chromosome 6 of the HLA genes and may have a role in PsA pathogenesis and consequently to therapeutically response. Rocha Loures et al. [18] conducted a study on as mixed Brazilian population, noticing that TNF-308, IL17A, and IL17F gene polymorphisms were associated with SpA and AS, while TNF-238, IL17A, and IL17F gene polymorphisms were associated with PsA, regardless of gender and HLA-B27.

Nevertheless, these authors recently conducted a meta-analysis with the aim of evaluating the effect of polymorphisms in the cytokines TNF, IL12B, IL23A, and IL23R on PsA risk. Researchers found an association between PsA with the following polymorphisms: TNF-238 G > A (rs361525), -308 G > A (rs1800629), and $-857 \mathrm{C}>\mathrm{T}$ (rs1799724); IL12B C > G (rs6887695) and A > C (rs3212227); IL23A A > G (rs2066808) and IL23R G > A (rs11209026) [19]. These findings do not only shed light on PsA pathogenesis, as they are useful to evaluate and may predict therapy response. During the last decade, many new biological immune modulators have entered the market. Biologics, including TNF-a inhibitors, are currently available for the treatment of immune-mediated or inflammatory diseases, such as rheumatoid arthritis, systemic lupus erythematosus, Crohn's disease, ankylosing spondylitis, systemic sclerosis, disseminated granuloma annulare, Ps and PsA [20]. Several polymorphisms have been proven to influence the response to these drugs. Etanercept (Enbrel, Pfizer, New York, NY, USA) is a dimeric fusion protein made by an extracellular portion of human soluble TNF- $\alpha$ receptor (r-TNF-p75) fused with the Fc portion of human immunoglobulin G1. Its efficacy has been validated not only among PsA patients but also 
among rheumatoid arthritis (RA), plaque psoriasis, spondyloarthritis, and juvenile idiopathic arthritis [21]. Indeed our group demonstrated that among PsA patients, single nucleotide polymorphisms (SNPs) $-308 \mathrm{G} / \mathrm{G},-857 \mathrm{C} / \mathrm{T},+489$ GG and GA, HLA-DRB1-encoding SE (allele ${ }^{\star} 0404$ and allele ${ }^{\star} 0101$ ) favored the response to etanercept, whereas SNP $-308 \mathrm{~A} / \mathrm{A}$ and TNFR1A AA reduced the response [22].

\section{Epigenetics}

The study of heritable changes occurring in gene expression which are not due to DNA sequence modifications is defined epigenetics [23]. As already known DNA is packed into chromatin and nucleosomes are its basic units. Each nucleosome consists of an octamer of histones. The latter, together with DNA sequence modifications are considered the two major epigenetics mechanisms. [24] Both types of epigenetic marks are transmitted stably through mitosis and act in concert to affect gene expression. A considerable change in DNA and histone modifications occur across loci, cells, and tissues within an organism [25]. As these changes can occur also in response to environmental stimuli, epigenetics represents the biological link between genes and the environment. It is now clear that epigenetics plays a key role in the etiology of many complex diseases including autoimmune and inflammatory disorders [26]. On these bases, researchers investigated the role of epigenetic upon Ps and PsA. Indeed, Pollock et al. [6] published a systematic review which analyzed the four major models of epigenetics which are parent-of-origin effect and genomic imprinting, DNA methylation, histone modifications and expression or activity of epigenetic modifying enzymes. Zhang et al. [14] demonstrated that the promoter methylation status and transcriptional activity of p16 and the colony-forming ability of high proliferative potential colony-forming cells (HPP-CFC) were related, suggesting that hematopoietic cells are involved in psoriasis pathogenesis. Moreover, significantly lower positive frequencies of promoter methylation and higher transcription levels for p15 and p21 genes were observed in Ps in comparison to normal volunteers. The lower promoter methylation of p15 and p21 genes may be an important mechanism for the dysfunctional growth regulation pathways in HPP-CFCs in Ps [27]. Furthermore, they also noticed that Ps pathogenesis was linked to bone marrow microenvironment changes, detecting elevated levels of the transcription protein HES1 mRNA, suggesting that Notch signaling pathway, which is a highly expressed regulatory mechanism is involved in Ps [28]. Regarding histones modifications, Ovejero-Benito et al. [24] investigated on their relationship with drug response therapy. They analyzed histones extracted from peripheral blood mononuclear cells (PBMCs) of 39 psoriasis patients treated with biological therapies before and after drug administration and 42 healthy subjects. Four histone modifications (H3 and $\mathrm{H} 4$ acetylation, H3K4 and H3K27 methylation) were determined by ELISA. However, Ps patients presented reduced levels of acetylated $\mathrm{H} 3$ and $\mathrm{H} 4$ and increased levels of methylated $\mathrm{H} 3 \mathrm{~K} 4$ 
compared to controls. In Ps patients without PsA, significant changes in methylated $\mathrm{H} 3 \mathrm{~K} 4$ were found between responders and non-responders to biological drugs at 3 and 6 months. Moreover, significant modifications in methylated H3K27 were found between responders and non-responders to biological drugs at 3 months.

\section{MicroRNAs}

MicroRNAs (miRNAs) are small molecules which act as post-transcriptional regulators by targeting the 3' untranslated region (3'UTR) of specific messenger RNAs (mRNAs) for degradation of translation repression [29]. Deletion studies demonstrated that they play a crucial role in immune cell development, especially under conditions of environmental stress such as infectious challenge and aging. However, miRNA relevance has been evaluated both upon innate and adaptive immunity [30]. Moreover, recent studies highlighted that miRNAs are highly expressed in Treg cells. Indeed, the expression of Foxp3 is controlled by miRNAs and a range of miRNAs are involved in the regulation of immunity, leading to the hypothesis that miRNAs abnormal expression may regulate the immune system with regards to chronic inflammatory disorders and cancers [31]. Indeed, as reported by Najm et al. [32] mi-RNAs play key roles in fibroblast-like synoviocytes migration, proliferation, apoptosis, and cytokine production, in macrophages polarization, as well as in $\mathrm{B}$ cells and $\mathrm{T}$ cell proliferation and differentiation, with a special emphasis on Treg/Th17 imbalance.

On this background, Lunardi et al. [33] published an original article in order to evaluate miRNA expression profiles in active and non-active PsA patients as compared to healthy controls. They performed a miRNA microarray analysis on PBMCs of PsA patients at the active stage and in the remission phase of the disease. They noticed that, among the modulated miRNAs, miR-126-3p was downregulated in active PsA patients, whereas its expression was higher in nonactive patients. Indeed, miR-126 overexpression induced a decrease of putative molecular targets with a potential role in PsA pathogenesis.

\section{Microbiome}

As above mentioned, Ps and PsA development depend both on genetic and environmental factors. Among the latter, researchers recently investigated the role of intestinal microbiome upon these conditions. However, the microbial communities that live in our gastrointestinal tract, termed the gut microbiota, are well known to play a fundamental role in many host processes, including immune system regulation [34]. Several authors reported the modulatory effects of gut microbiota on immune cells functioning. Indeed, gut mucosa is inhabited by different $\mathrm{T}$ lymphocytes, including regulatory $\mathrm{T}$ cells [35] [36]. It was hypothesized that pro-inflammatory responses initiated in gut mucosa could contribute to the induction and progression of autoimmune conditions affecting several systems, including skin and joint inflammatory diseases [37]. Furthermore, sev- 
eral studies demonstrated that the intestinal microbiota is altered in subjects with spondyloarthritis [38] [39].

In order to characterize the diversity and taxonomic relative abundance of the gut microbiota in PsA patients, Scher et al. [40] conducted a study among recent-onset PsA and Ps patients compared to healthy controls. Authors reported that PsA and Ps patients had a lower relative abundance of multiple intestinal bacteria. Although some genera were concomitantly decreased in both conditions, PsA samples had a lower abundance of reportedly beneficial taxa. Notably, this gut microbiota profile in PsA was similar to IBD patients. Moreover, Chen et al. [41] investigated the intestinal microbiota and the potential genetic function in relation to clinical presentation in Ps patients. They demonstrated that distinct fecal microbial composition in Ps might be associated with altered transport of carbohydrate, cobalamin, and iron, as well as chemotaxis, predicting metabolic dysregulation in Ps patients. In order to find new effective and more tailored therapies, Kragsnaes et al. [42] elaborated a randomized, placebo-controlled trial of fecal microbiota transplantation (FMT) infused into the small intestine of patients with PsA with active peripheral disease who are non-responsive to methotrexate (MTX). This study started in December 2017 and is still ongoing. Nevertheless, Selvanderan et al. [43] reported that fecal microbiota transplantation for Clostridium difficile infection resulting in a decrease in PsA disease activity.

\section{Conclusion}

PsA is a heterogeneous disease and clinicians often have to face several comorbidities, such as cardiovascular disease, obesity, uveitis, depression, and inflammatory bowel disease. Therefore, a multidisciplinary approach is recommended [44]. Early identification leading to timely diagnosis and treatment is crucial to avoid long-term structural damage and disability and the associated socioeconomic consequences. The increase in therapeutic options, such as disease-modifying antirheumatic drugs, both biological and targeted synthetic, has revolutionized the treatment of skin and joint disease and has prompted clinicians to use the full clinical picture of an individual patient to make rational treatment decisions [45]. Several efforts have been made to understand Ps and PsA pathogenesis in order to improve early diagnosis and more tailored therapies. Further studies on genetic factors and epigenetics will give new insights into these complex disorders.

\section{Conflicts of Interest}

The authors declare no conflicts of interest regarding the publication of this paper.

\section{References}

[1] Stephen, D.D. (2017) Psoriatic Arthritis. Australasian Radiology, 1, 80-82. https://doi.org/10.1111/j.1440-1673.1957.tb00848.x

[2] Moll, J.M.H. and Wright, V. (1973) Psoriatic Arthritis. Seminars in Arthritis and Rheumatism, 3, 55-78. https://doi.org/10.1016/0049-0172(73)90035-8

[3] Solmaz, D., Eder, L. and Aydin, S.Z. (2018) Update on the Epidemiology, Risk Fac- 
tors, and Disease Outcomes of Psoriatic Arthritis. Best Practice \& Research Clinical Rheumatology, 32, 295-311. https://doi.org/10.1016/j.berh.2018.09.006

[4] Tang, C.-H. (2019) Research of Pathogenesis and Novel Therapeutics in Arthritis. International Journal of Molecular Sciences, 20, 1646.

https://doi.org/10.3390/ijms20071646

[5] Filaci, G., Rizzi, M., Setti, M., et al. (2005) Non-Antigen-Specific CD8+ T Suppressor Lymphocytes in Diseases Characterized by Chronic Immune Responses and Inflammation. Annals of the New York Academy of Sciences, 1050, 115-123. https://doi.org/10.1196/annals.1313.013

[6] Pollock, R.A., Abji, F. and Gladman, D.D. (2017) Epigenetics of Psoriatic Disease: A Systematic Review and Critical Appraisal. Journal of Autoimmunity, 78, 29-38. https://doi.org/10.1016/j.jaut.2016.12.002

[7] Moll, J.M.H. and Wright, V. (1973) Familial Occurrence of Psoriatic Arthritis. Annals of the Rheumatic Diseases, 32, 181-201. https://doi.org/10.1136/ard.32.3.181

[8] Chandran, V., Schentag, C.T., Brockbank, J.E., et al. (2009) Familial Aggregation of Psoriatic Arthritis. Annals of the Rheumatic Diseases, 68, 664-667.

https://doi.org/10.1136/ard.2008.089367

[9] Kimball, A.B., Leonardi, C., Stahle, M., et al. (2014) Demography, Baseline Disease Characteristics and Treatment History of Patients with Psoriasis Enrolled in a Multicentre, Prospective, Disease-Based Registry (PSOLAR). British Journal of Dermatology, 171, 137-147. https://doi.org/10.1111/bjd.13013

[10] Roudier, J., Massy, E. and Balandraud, N. (2018) Diagnostic Contribution of HLA-A,B,C,DR Genotyping in Inflammatory Joint Disease. Joint Bone Spine, 85, 511-513. https://doi.org/10.1016/j.jbspin.2018.02.007

[11] Tsoi, L.C., Spain, S.L., Knight, J., et al. (2012) Identification of 15 New Psoriasis Susceptibility Loci Highlights the Role of Innate Immunity. Nature Genetics, 44, 1341-1348. https://doi.org/10.1038/ng.2467

[12] Genetic Analysis of Psoriasis Consortium and the Wellcome Trust Case Control Consortium 2 (2010) A Genome-Wide Association Study Identifies New Psoriasis Susceptibility Loci and an Interaction between $H L A-C$ and ERAP1. Nature Genetics, 42, 985-990. https://doi.org/10.1038/ng.694

[13] Das, A., Chandra, A., Chakraborty, J., et al. (2017) Associations of ERAP1 Coding Variants and Domain Specific Interaction with $H L A-C * 06$ in the Early Onset Psoriasis Patients of India. Human Immunology, 78, 724-730.

https://doi.org/10.1016/j.humimm.2017.08.006

[14] Fan, X., Yang, S., Sun, L.D., et al. (2007) Comparison of Clinical Features of HLA-Cw ${ }^{\star} 0602$-Positive and -Negative Psoriasis Patients in a Han Chinese Population. Acta Dermato- Venereologica, 87, 335-340. https://doi.org/10.2340/00015555-0253

[15] Jenisch, S., Henseler, T., Nair, R.P., et al. (2002) Linkage Analysis of Human Leukocyte Antigen (HLA) Markers in Familial Psoriasis: Strong Disequilibrium Effects Provide Evidence for a Major Determinant in the HLA-B/-C Region. The American Journal of Human Genetics, 63, 191-199. https://doi.org/10.1086/301899

[16] Gudjonsson, J.E., Karason, A., Runarsdottir, E.H., et al. (2006) Distinct Clinical Differences between HLA-Cw*0602 Positive and Negative Psoriasis Patients-An analysis of 1019 HLA-C- and HLA-B-Typed Patients. Journal of Investigative Dermatology, 126, 740-745. https://doi.org/10.1038/sj.jid.5700118

[17] Dand, N., Duckworth, M., Baudry, D., et al. (2019) $H L A-C^{\star} 06: 02$ Genotype Is a Predictive Biomarker of Biologic Treatment Response in Psoriasis. Journal of Al- 
lergy and Clinical Immunology, 143, 2120-2130. https://doi.org/10.1016/j.jaci.2018.11.038

[18] Rocha Loures, M.A., Macedo, L.C., Reis, D.M., et al. (2018) Influence of TNF and IL17 Gene Polymorphisms on the Spondyloarthritis Immunopathogenesis, Regardless of HLA-B27, in a Brazilian Population. Mediators of Inflammation, 2018, Article ID: 1395823. https://doi.org/10.1155/2018/1395823

[19] Loures, M.A.R., Alves, H.V., de Moraes, A.G., et al. (2019) Association of TNF, $I L 12$, and IL23 Gene Polymorphisms and Psoriatic Arthritis: Meta-Analysis. Expert Review of Clinical Immunology, 15, 303-313. https://doi.org/10.1080/1744666X.2019.1564039

[20] Murdaca, G., Gulli, R., Spanò, F., et al. (2014) TNF- $\alpha$ Gene Polymorphisms: Association with Disease Susceptibility and Response to Anti-TNF- $\alpha$ Treatment in Psoriatic Arthritis. Journal of Investigative Dermatology, 134, 2503-2509. https://doi.org/10.1038/jid.2014.123

[21] Guillot, X., Prati, C., Sondag, M. and Wendling, D. (2017) Etanercept for Treating Axial Spondyloarthritis. Expert Opinion on Biological Therapy, 17, 1173-1181. https://doi.org/10.1080/14712598.2017.1347156

[22] Murdaca, G., Spanò, F., Contatore, M., Guastalla, A., Magnani, O. and Puppo, F. (2014) Pharmacogenetics of Etanercept: Role of TNF- $\alpha$ Gene Polymorphisms in Improving Its Efficacy. Expert Opinion on Drug Metabolism \& Toxicology, 10, 1703-1710. https://doi.org/10.1517/17425255.2014.970165

[23] Eccleston, A., DeWitt, N., Gunter, C., Marte, B. and Nath, D. (2007) Epigenetics. Nature, 447, 395.

[24] Ovejero-Benito, M.C., Reolid, A., Sánchez-Jiménez, P., et al. (2018) Histone Modifications Associated with Biological Drug Response in Moderate-to-Severe Psoriasis. Experimental Dermatology, 27, 1361-1371. https://doi.org/10.1111/exd.13790

[25] Harvey, Z.H., Chen, Y. and Jarosz, D.F. (2018) Protein-Based Inheritance: Epigenetics beyond the Chromosome. Molecular Cell, 69, 195-202. https://doi.org/10.1016/j.molcel.2017.10.030

[26] Tost, J., Gay, S. and Firestein, G. (2017) Epigenetics of the Immune System and Alterations in Inflammation and Autoimmunity. Epigenomics, 9, 371-373. https://doi.org/10.2217/epi-2017-0026

[27] Zhang, K., Zhang, R., Li, X., Yin, G. and Niu, X. (2009) Promoter Methylation Status of p15 and p21 Genes in HPP-CFCs of Bone Marrow of Patients with Psoriasis. European Journal of Dermatology, 19, 141-146. https://doi.org/10.1684/ejd.2008.0618

[28] Zhang, K., Hou, R., Niu, X., et al. (2010) Decreased Colony Formation of High Proliferative Potential Colony-Forming Cells and Granulocyte-Macrophage Colony-Forming Units and Increased Hes-1 Expression in Bone Marrow Mononuclear Cells from Patients with Psoriasis. British Journal of Dermatology, 163, 93-101. https://doi.org/10.1111/j.1365-2133.2010.09790.x

[29] Ha, T.-Y. (2011) The Role of MicroRNAs in Regulatory T Cells and in the Immune Response. Immune Network, 11, 11. https://doi.org/10.4110/in.2011.11.1.11

[30] Mehta, A. and Baltimore, D. (2016) MicroRNAs as Regulatory Elements in Immune System Logic. Nature Reviews Immunology, 16, 279-294. https://doi.org/10.1038/nri.2016.40

[31] Raisch, J., Darfeuille-Michaud, A. and Nguyen, H.T.T. (2013) Role of MicroRNAs in the Immune System, Inflammation and Cancer. World Journal of Gastroenterology, 19, 2985-2996. https://doi.org/10.3748/wjg.v19.i20.2985 
[32] Najm, A., Blanchard, F. and Le Goff, B. (2019) Micro-RNAs in Inflammatory Arthritis: From Physiopathology to Diagnosis, Prognosis and Therapeutic Opportunities. Biochemical Pharmacology, 165, 134-144. https://doi.org/10.1016/j.bcp.2019.02.031

[33] Pelosi, A., Lunardi, C., Fiore, P.F., et al. (2018) MicroRNA Expression Profiling in Psoriatic Arthritis. BioMed Research International, 2018, Article ID: 7305380. https://doi.org/10.1155/2018/7305380

[34] Chimenti, M.S., Perricone, C., Novelli, L., et al. (2018) Interaction between Microbiome and Host Genetics in Psoriatic Arthritis. Autoimmunity Reviews, 17, 276-283. https://doi.org/10.1016/j.autrev.2018.01.002

[35] Gilis, E., Mortier, C., Venken, K., Debusschere, K., Vereecke, L. and Elewaut, D. (2018) The Role of the Microbiome in Gut and Joint Inflammation in Psoriatic Arthritis and Spondyloarthritis. The Journal of Rheumatology, 94, 36-39.

[36] Eppinga, H., Thio, H.B., Peppelenbosch, M.P. and Konstantinov, S.R. (2014) The Gut Microbiome Dysbiosis and Its Potential Role in Psoriatic Arthritis. International Journal of Clinical Rheumatology, 9, 559-565.

[37] Lynch, S.V. and Pedersen, O. (2016) The Human Intestinal Microbiome in Health and Disease. The New England Journal of Medicine, 375, 2369-2379. https://doi.org/10.1056/NEJMra1600266

[38] Stoll, M.L. (2014) Gut Microbes, Immunity, and Spondyloarthritis. Clinical Immunology, 159, 134-142. https://doi.org/10.1016/j.clim.2015.05.001

[39] Ragab, G., Atkinson, T.P. and Stoll, M.L. (2018) The Microbiome in Rheumatic Diseases and Infection. Springer Nature, Switzerland. https://doi.org/10.1007/978-3-319-79026-8

[40] Scher, J.U., Ubeda, C., Artacho, A., et al. (2015) Decreased Bacterial Diversity Characterizes an Altered Gut Microbiota in Psoriatic Arthritis and Resembles Dysbiosis of Inflammatory Bowel Disease. Arthritis \& Rheumatology, 67, 128-139. https://doi.org/10.1002/art.38892

[41] Chen, Y.J., Ho, H.J., Tseng, C.H., Lai, Z.L., Shieh, J.J. and Wu, C.Y. (2018) Intestinal Microbiota Profiling and Predicted Metabolic Dysregulation in Psoriasis Patients. Experimental Dermatology, 27, 1336-1343. https://doi.org/10.1111/exd.13786

[42] Kragsnaes, M.S., Kjeldsen, J., Horn, H.C., et al. (2018) Efficacy and Safety of Faecal Microbiota Transplantation in Patients with Psoriatic Arthritis: Protocol for a 6-Month, Double-Blind, Randomised, Placebo-Controlled Trial. BMJ Open, 8, e019231. https://doi.org/10.1136/bmjopen-2017-019231

[43] Selvanderan, S.P., Goldblatt, F., Nguyen, N.Q. and Costello, S.P. (2019) Faecal Microbiota Transplantation for Clostridium Difficile Infection Resulting in a Decrease in Psoriatic Arthritis Disease Activity. Clinical and Experimental Rheumatology, 37, 514-515.

[44] The Lancet (2018) Psoriatic Arthritis: Classification and Holistic Management. The Lancet, 391, 2185. https://doi.org/10.1016/S0140-6736(18)31249-2

[45] Van den Bosch, F. and Coates, L. (2018) Clinical Management of Psoriatic Arthritis. The Lancet, 391, 2285-2294. https://doi.org/10.1016/S0140-6736(18)30949-8 